\title{
Umbilical cord-derived mesenchymal stem cells inhibit growth and promote apoptosis of HepG2 cells
}

\author{
YING-MEI TANG ${ }^{1}$, WEI-MIN BAO ${ }^{2}$, JIN-HUI YANG ${ }^{1}$, LIN-KUN MA $^{3}$, JING YANG $^{1}$, YING XU $^{1}$, \\ LI-HONG YANG ${ }^{1}$, FENG SHA ${ }^{1}$, ZHI-YUAN XU ${ }^{1}$, HUA-MEI WU ${ }^{1}$, WEI ZHOU ${ }^{1}$, YAN LI ${ }^{1}$ and YU-HUA LI ${ }^{1}$ \\ ${ }^{1}$ Department of Gastroenterology, The 2nd Affiliated Hospital of Kunming Medical University, \\ Yunnan Research Center for Liver Diseases, Kunming, Yunnan 650033; \\ ${ }^{2}$ Department of General Surgery, Yunnan Provincial 1st People's Hospital, Kunming, Yunnan 650032; \\ ${ }^{3}$ Department of Ophthamology, The 2nd Affiliated Hospital of Kunming Medical University, \\ Kunming, Yunnan 650033, P.R. China
}

Received May 25, 2015; Accepted May 23, 2016

DOI: $10.3892 / \mathrm{mmr} .2016 .5537$

\begin{abstract}
Hepatocellular carcinoma is the fifth most common type of cancer worldwide and remains difficult to treat. The aim of this study was to investigate the effects of mesenchymal stem cells (MSCs) derived from the umbilical cord (UC-MSCs) on HepG2 hepatocellular carcinoma cells. UC-MSCs were co-cultured with HepG2 cells and biomarkers of UC-MSCs were analyzed by flow cytometry. mRNA and protein expression of genes were determined by reverse transcription-polymerase chain reaction and flow cytometry, respectively. Passage three and seven UC-MSCs expressed CD29, CD44, CD90 and CD105, whereas CD34 and CD45 were absent on these cells. Co-culture with UC-MSCs inhibited proliferation and promoted apoptosis of HepG2 cells in a time-dependent manner. The initial seeding density of UC-MSCs also influenced the proliferation and apoptosis of HepG2 cells, with an increased number of UC-MSCs causing enhanced proliferation inhibition and cell apoptosis. Co-culture with UC-MSCs downregulated mRNA and protein expression of $\alpha$-fetoprotein (AFP), Bcl-2 and Survivin in HepG2 cells. Thus, UC-MSCs may inhibit growth and promote apoptosis of HepG2 cells through downregulation of AFP, Bcl-2 and Survivin. US-MSCs may be used as a novel therapy for treating hepatocellular carcinoma in the future.
\end{abstract}

Correspondence to: Dr Jin-Hui Yang, Department of Gastroenterology, The 2nd Affiliated Hospital of Kunming Medical University, Yunnan Research Center for Liver Diseases, 374 Dianmian Road, Kunming, Yunnan 650033, P.R. China E-mail: tangyingmei_med@163.com; tangyingmei9695@163.com

Key words: umbilical cord-derived mesenchymal stem cells, HepG2 cells, co-culture, proliferation, apoptosis

\section{Introduction}

Hepatocellular carcinoma (HCC), which accounts for 80-90\% of primary liver cancer, is the fifth most common type of cancer worldwide and the third most common cause of cancer-related mortality (1). Liver resection is currently the first line of treatment in non-cirrhotic patients, yet the reported overall 5 -year survival after HCC liver resection is $30-60 \%$, with a high incidence of recurrence (50-80\%) (1-5). Currently, there are no widely accepted chemopreventive strategies to limit the progression of HCC once liver cirrhosis is established (6). Liver transplantation provides a good outcome for patients with HCC meeting the Milan criteria (single nodule $\leq 5 \mathrm{~cm}$ or 2 or three nodules $\leq 3 \mathrm{~cm}$ ), and leads to 5 -year survival rates of $70 \%$ and low recurrence rates (7). The application of liver transplantation is rare, however, due to a limited source of liver donors, high cost and organ rejection following transplantation.

Adult and embryonic hepatocytes, hepatic stem/progenitor cells and extrahepatic stem cells have been used as transplantable cell sources for liver regeneration (8). Such cellular therapy may provide a novel approach for the treatment of advanced stages of HCC. Umbilical cord mesenchymal stem cells (UC-MSCs) are a subgroup of MSCs that possess the potential to differentiate into several mesodermal tissues (bone, cartilage, tendon, muscle and adipose tissue) (9), endodermal tissue (hepatocytes) (10), and ectodermal tissue (neurons) $(11,12)$. However, the effects of MSCs on tumor cells remain controversial. Li et al (13) demonstrated that MSCs enhanced tumor growth but significantly inhibited the invasiveness and metastasis of HCC. However, another group demonstrated that MSCs possess intrinsic antineoplastic properties in a Kaposi's sarcoma model (14). A recent study indicated that human UC-MSCs significantly inhibited the growth of breast cancer stem cells (CSCs) in vitro and in vivo (15).

Based on these data, it was hypothesize that UC-MSCs may have antitumor effects on cancer cells, such as HepG2 HCC cells. The present study investigated the effects of UC-MSCs on HepG2 cells using a Transwell co-culture approach. The results provide valuable insights into the biological 
characteristics of UC-MSCs and provide evidence for their potential therapeutic use for the treatment of HCC.

\section{Materials and methods}

Reagents. Fetal bovine serum (FBS), Dulbecco's modified Eagle's medium (DMEM)/F12, 0.25\% trypsin and phosphate-buffered saline (PBS) were purchased from GE Healthcare (Logan, UT, USA). Fluorescein isothiocyanate-conjugated (FITC) mice monoclonal antibodies, including anti-CD29-FITC (cat no. 6604105; 1:50), anti-CD34-FITC (cat. no. IM1870; 1:50), anti-CD44-FITC (cat. no. IM1219U; 1:50), anti-CD45-FITC (cat. no. IM0782U; 1:50), anti-CD90-FITC (cat. no. IM1839U; 1:50), anti-CD105-PE (cat. no. B76299; 1:50), as well as intra-1 (cat. no. A07803; 1:50) and intra-2 (cat.no. A07803; 1:50) were obtained from Beckman Coulter, Inc. (Brea, CA, USA). Monoclonal phycoerythrin (PE)-conjugated antibodies, anti-Bcl-2-PE (cat. no. A15796; 1:50; Invitrogen, Thermo Fisher Scientific, Inc., Waltham, MA, USA) and anti-Survivin-PE (cat. no. 129176; 1:50; eBioscience, Inc., San Diego, CA, USA). Anti- $\alpha$ fetoprotein (AFP) primary antibody and FITC-conjugated $\operatorname{IgG}(\mathrm{H}+\mathrm{L})$ antibody were provided by LsBio (Seattle, WA, USA) and Invitrogen, Thermo Fisher Scientific Inc., respectively. An RNA extraction kit was purchased from TianGen BioTech Co., Ltd. (Beijing, China). An RNA reverse transcription reaction kit and RNA quantification kit were purchased from Bio-Rad (Hercules, CA, USA). Primers were synthesized by Sangon Biotech Co., Ltd. (Shanghai, China).

Cell culture. Third passage human UC-MSCs were obtained from Beike Biotechnology (Shenzhen, China). These cells were obtained from informed, healthy donors after normal spontaneous vaginal deliveries. The procedure for cell collection and mononuclear cell extraction, cultivation and harvest, has been reported in a previous publication (16). Human HCC HepG2 cells were kindly provided by Professor Lin Wang (17) from the Department of Hepatobiliary Surgery, The 2nd Affiliated Hospital of Kunming Medical University (Kunming, China). UC-MSCs and HepG2 cells were maintained in DMEM/F12 containing 10\% FBS, $100 \mathrm{U} / \mathrm{ml}$ penicillin and $100 \mu \mathrm{g} / \mathrm{ml}$ streptomycin (GE Healthcare Life Sciences, Logan, UT, USA). Cells were incubated in a $5 \%$ $\mathrm{CO}_{2}$-humidified incubator at $37^{\circ} \mathrm{C}$. The culture medium was changed every two or three days. When cells reached 80-90\% confluency, cell passage was conducted using $0.25 \%$ trypsin for digestion. Passage seven to eight UC-MSCs were used for the following experiments.

Co-culture procedure. For co-culture of UC-MSCs with HepG2 cells, a Transwell co-culture system (pore size, $0.4 \mu \mathrm{m}$; Corning, Corning, NY, USA) was utilized. UC-MSCs were collected by trypsin digestion, resuspended in fresh culture medium, adjusted to the appropriate cell density $\left(2.5 \times 10^{5}-4 \times 10^{6}\right.$ cells/well $)$ and seeded onto a six-well plate ( $2 \mathrm{ml}$ cell suspension for each well). After the initial seeding of UC-MSCs (24 h), 1 x10 ${ }^{6} \mathrm{HepG} 2$ cells suspended in $2 \mathrm{ml}$ culture medium were seeded into the upper compartment of the Transwell. Cell morphology was observed under a phase contrast microscopy (BX53, Olympus, Tokyo, Japan).
Flow cytometric analysis of UC-MSC surface biomarkers. Passage three and passage seven UC-MSCs were used for cell identification. Cells were washed with PBS and digested with $0.25 \%$ trypsin. After centrifuging at $300 \mathrm{x}$ g for $5 \mathrm{~min}$, the supernatant was removed and cells were washed twice with PBS. Cells were then incubated with FITC-conjugated primary antibodies against CD29, CD34, CD44, CD45, CD 90 or CD105 for $30 \mathrm{~min}$ at room temperature in the dark. The expression of cell surface biomarkers was determined using flow cytometric analysis. Fluorescence acquisition was performed in a Coulter-EPICS XL flow cytometer (Beckman-Coulter, Inc.) with a $488 \mathrm{~nm}$ argon-ion laser.

Evaluation of cell proliferation. After 24, 48 or $72 \mathrm{~h}$ of co-culture, HepG2 cells were collected and seeded onto a 96-well plate. Six wells were used for each treatment. Cells were maintained with the culture medium collected from the co-culture system. After $24 \mathrm{~h}$, the co-culture medium was removed and replaced with $180 \mu \mathrm{l}$ fresh culture medium. Then, $20 \mu 1$ 3-(4,5-dimethylthiazol-2-yl)-2,5-diphenyltetrazolium bromide (MTT; $5 \mathrm{mg} / \mathrm{ml}$; Beckman-Coulter) was added to each well. After an additional $4 \mathrm{~h}$ of incubation at $37^{\circ} \mathrm{C}$, the medium was removed and $150 \mu \mathrm{l}$ dimethyl sulfoxide was added to each well to resuspend the MTT metabolic product. The absorbance of the dissolved formazan was measured at $490 \mathrm{~nm}\left(\mathrm{~A}_{490}\right)$ using a scanning microplate spectrophotometer (Bio-Rad). The proliferation inhibition rate was calculated using the following formula: Proliferation inhibition rate = $\left(\mathrm{A}_{490, \text { Control }}-\mathrm{A}_{490, \text { Sample }}\right) / \mathrm{A}_{490, \text { Control }} \mathrm{x} 100$.

Measurement of cell apoptosis. Cell apoptosis was measured by flow cytometry and confirmed by terminal deoxynucleotidyl transferase (TdT)-mediated nick end labeling (TUNEL). Using the Transwell system, UC-MSCs were co-cultured with HepG2 cells. A range of initial seeding densities for UC-MSCs was tested $\left(0.25 \times 10^{6}, 0.5 \times 10^{6}, 1 \times 10^{6}, 2 \times 10^{6}\right.$ or $4 \times 10^{6}$ cells/well). After co-culture, HepG2 cells were collected and cell density was adjusted to $1 \times 10^{6}$ cells $/ \mathrm{ml}$. After washing with PBS, cells were fixed with $4 \%$ paraformaldehyde (Sigma-Aldrich, St. Louis, MO, USA) for $30 \mathrm{~min}$ at $4^{\circ} \mathrm{C}$. Then, cells were washed with $0.2 \%$ bovine serum albumin (BSA; GE Healthcare Life Sciences) in PBS and incubated with $70 \%$ ethanol at $20^{\circ} \mathrm{C}$ for an additional $30 \mathrm{~min}$. After washing twice with $0.2 \%$ BSA in PBS, cells were treated with the TdT solution $(1.5 \mu \mathrm{l} \mathrm{TdT}$, $27 \mu 1$ TdT buffer and 1.5 $\mu 1$ FITC-dUTP; GE Healthcare Life Sciences) or a negative control solution ( $28.5 \mu \mathrm{l}$ TdT buffer and $1.5 \mu 1$ FITC-dUTP) for $1 \mathrm{~h}$ at $37^{\circ} \mathrm{C}$. After washing, samples were incubated with $20 \mu \mathrm{l}$ of $10 \mathrm{mg} / \mathrm{ml}$ RNase (Sigma-Aldrich) and $20 \mu \mathrm{l}$ of $0.1 \%$ Triton X-100 (Invitrogen; Thermo Fisher Scientific, Inc.) for $15 \mathrm{~min}$ at $37^{\circ} \mathrm{C}$ followed by $100 \mu \mathrm{g} / \mathrm{ml}$ propidium iodide (PI; Sigma-Aldrich) staining for 15-30 min. Fluorescence acquisition and analysis were performed using a flow cytometer (Beckman-Coulter, Inc.).

Reverse transcription-quantitative polymerase chain reaction $(R T-q P C R)$. UC-MSCs were seeded and co-cultured with HepG2 cells for $72 \mathrm{~h}$ with a range of initial seeding densities $\left(0.5 \times 10^{6}, 0.75 \times 10^{6}, 1 \times 10^{6}, 1.25 \times 10^{6}\right.$ or $1.5 \times 10^{6}$ cells/well $)$. Total RNA was extracted from HepG2 cells using an RNA extraction kit according to the manufacturer's instructions. RNA 
A

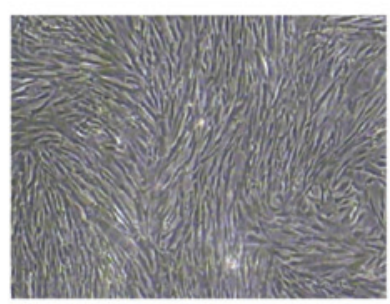

B

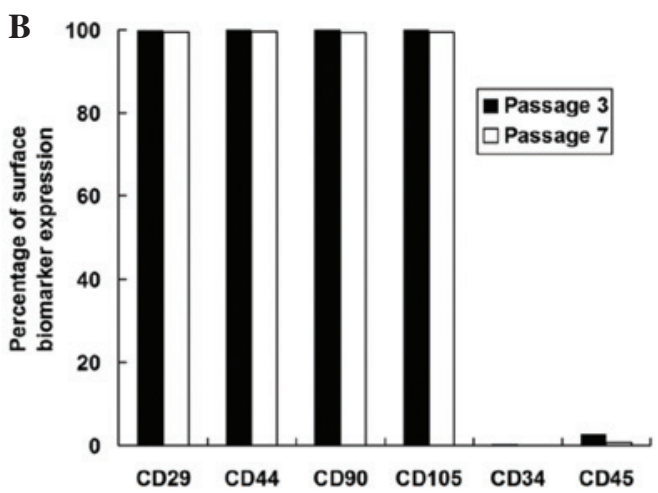

C
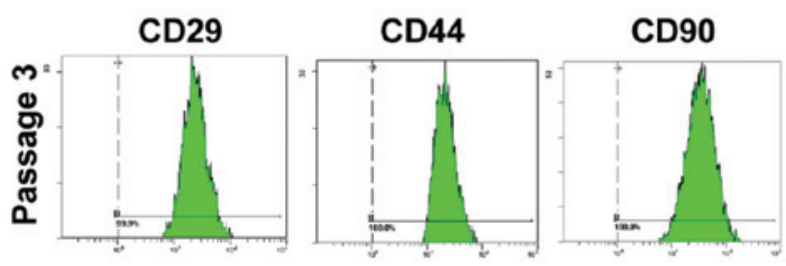
CD105

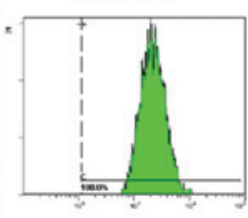

CD34 CD45
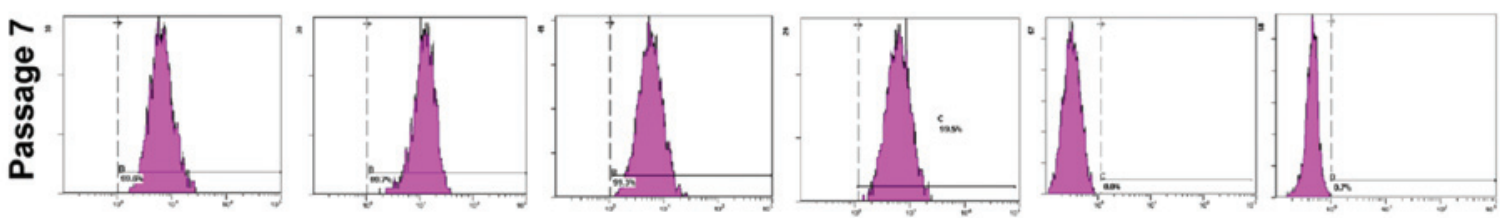

Figure 1. Morphological and biological characteristics of UC-MSCs. (A) Light micrograph of in vitro cultured UC-MSCs. Original magnification, x40. (B) The expression of surface biomarkers in passage 3 or passage 7 UC-MSCs. The cell surface expression of CD29, CD44, CD90, CD105, CD34 and CD45 were evaluated by flow cytometric analysis using specific antibodies. No significant difference was found in the expression of biomarkers between passage three and passage seven UC-MSCs. (C) Representative flow cytometric data. The X-axis indicates the fluorescence channel and the Y-axis depicts the number of cells. UC-MSCs, umbilical cord-derived mesenchymal stem cells.

concentration and purity were assayed by gel electrophoresis. Total RNA $(1 \mu \mathrm{g})$ was reverse transcribed using a reverse transcription kit according to the manufacturer's instructions. PCR amplification was conducted with specific primers designed by Primer 5.0 software (PREMIER Biosoft, Palo Alto, CA, USA) as follows: Forward: 5'-TGCGTTTCT CGTTGCTTACA-3' and reverse: 5'-GCTGCCATTTTTCTG GTGAT-3' for AFP; forward: 5'-GTGGATGACTGAGTACCT GAACC-3' and reverse: 5'-AGACAGCCAGGAGAAATC AAAC-3' for BCL2 gene; forward: 5'-GACCACCGCATC TCTACATTC-3' and reverse: 5'-AAGTCTGGCTCGTTC TCAGTG-3' for baculoviral IAP repeat containing fifth (BIRC5) gene encoding survivin; and RPS13 was used as a housekeeping gene forward: 5'-GTTGCTGTTCGAAAGCAT CTTG-3' and reverse: 5'-AATATCGAGCCAAACGGT GAA-3'. The PCR reactions were heated to $94^{\circ} \mathrm{C}$ for $5 \mathrm{~min}$, and subjected to 30 cycles of $94^{\circ} \mathrm{C}$ for $30 \mathrm{sec}, 60^{\circ} \mathrm{C}$ for $30 \mathrm{sec}$ and $72^{\circ} \mathrm{C}$ for $45 \mathrm{sec}$. Each experimental condition was repeated in triplicate. The amplified product lengths were 81, 124 and $116 \mathrm{bp}$ for AFP, Bcl-2 and Survivin, respectively. The relative expression from amplified RNA samples was calculated using the $2^{-\Delta \Delta \mathrm{Cq}}$ method (18) using Applied Biosystems 7500 thermocycler 2.3 (Thermo Fisher Scientific, Inc.).

Evaluation of protein expression. UC-MSCs were seeded and co-cultured with HepG2 cells for $72 \mathrm{~h}$ with a range of initial seeding densities $\left(0.25 \times 10^{6}, 0.5 \times 10^{6}, 1 \times 10^{6}, 2 \times 10^{6}\right.$ or $4 \times 10^{6}$ cells/well). HepG2 cells were collected and incubated with $0.5 \%$ Tween-20, intra- 1 and intra- 2 for membrane permeability. Cells were treated with $20 \mu \mathrm{l}$ AFP-FITC, Bcl-2-PE or Survivin-PE antibodies. The primary antibody $(20 \mu \mathrm{l})$ was added to tube one, washed with PBS after $30 \mathrm{~min}$, then FITC $(\mathrm{H}+\mathrm{L}) \mathrm{IgG}$ was added. Concurrently, $20 \mu \mathrm{lg} \mathrm{Ig}$-FITC was added into tube two as negative control. After washing three times with a $0.5 \%$ Tween-20 solution, fluorescence intensities were analyzed with a flow cytometer (Beckman-Coulter).

Statistical analysis. Data were analyzed using SPSS 16.0 software (SPSS Inc., Chicago, IL, USA). Statistical significance was determined using repeated measures analysis of variance, a Bonferroni correction was then performed as a post-hoc test. Data are presented as the mean \pm standard deviation. $\mathrm{P}<0.05$ was considered to indicate a statistically significant difference.

\section{Results}

In vitro culture and identification of UC-MSCs. In vitro cultured UC-MSCs exhibited a spindle-like structure and were arranged in parallel or in swirls (Fig. 1A). These morphological characteristics could be maintained until passage 20 (data not shown), which is consistent with the results of previous studies $(19,20)$. Using flow cytometric analysis, the cell surface expression of stem cell biomarkers, CD29, CD44, CD90, CD105, CD34 and CD45 were determined. As shown in (Fig. 1B and C), UC-MSCs expressed CD29, CD44, CD90 and CD105 but lacked CD34 and CD45, confirming the 
A

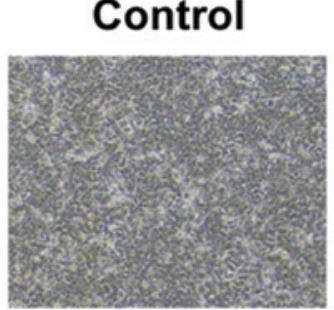

$1 \times 10^{6}$

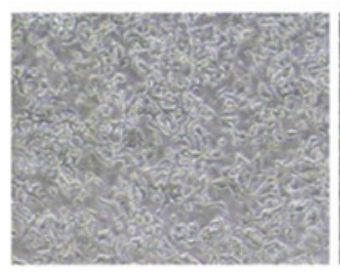

$0.5 \times 10^{6}$

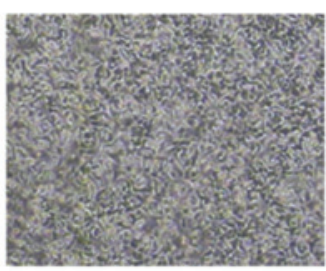

$1.25 \times 10^{6}$

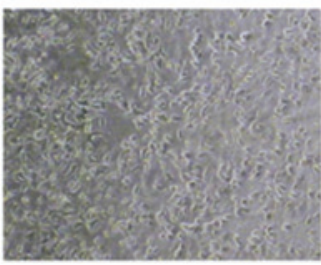

$0.75 \times 10^{6}$

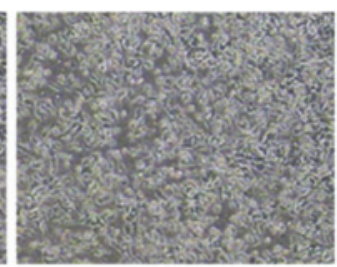

$1.5 \times 10^{6}$

B

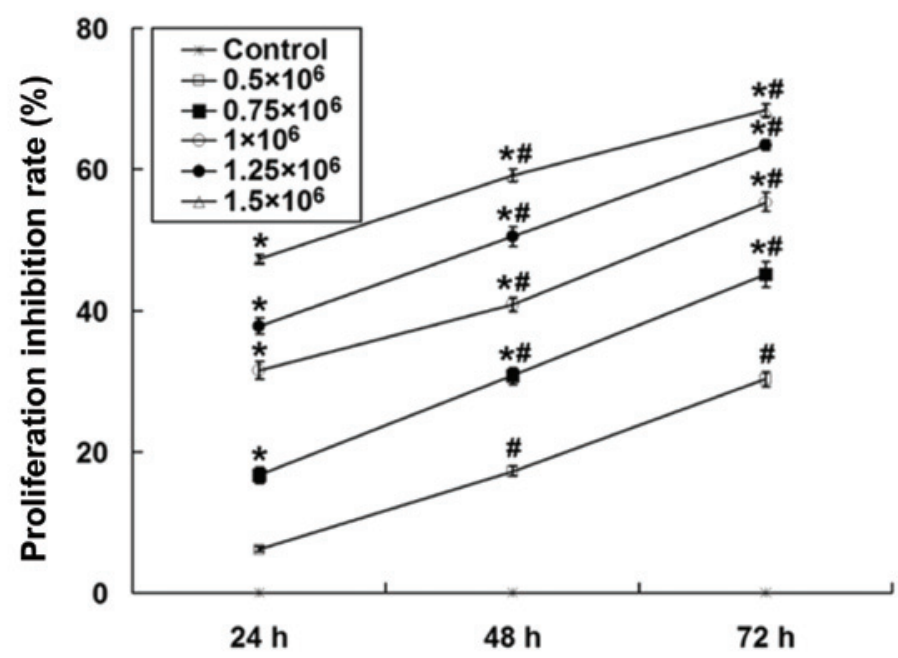

Figure 2. UC-MSCs inhibited the proliferation of HepG2 cells after co-culture. Using a Transwell system, UC-MSCs were co-cultured with HepG2 cells. A range of initial seeding densities for UC-MSCs was tested. (A) Representative light micrographs of HepG2 cells after co-culture with $0\left(\right.$ control), $0.5 \times 10^{6}, 0.75 \times 10^{6}, 1 \times 10^{6}$, $1.25 \times 10^{6}$, or $1.5 \times 10^{6} \mathrm{UC}-\mathrm{MSCs}$ per well for $72 \mathrm{~h}$. Magnification, $\mathrm{x} 40$. (B) The proliferation inhibition rate was calculated from three independent experiments. The $\mathrm{x}$-axis indicates the incubation time (h). ${ }^{*} \mathrm{P}<0.01$ compared with $0.5 \times 10^{6} \mathrm{UC}$-MSCs incubation; ${ }^{*} \mathrm{P}<0.01$ compared with $24 \mathrm{~h}$ incubation. UC-MSCs, umbilical cord-derived mesenchymal stem cells.

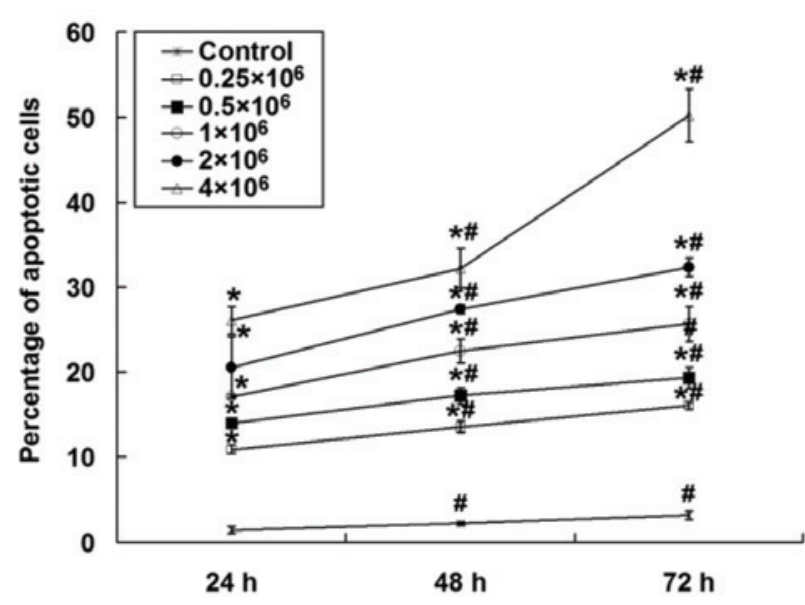

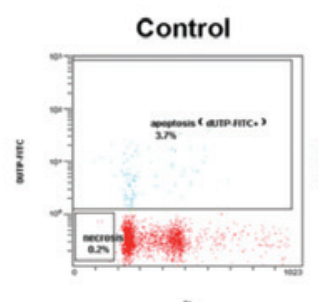
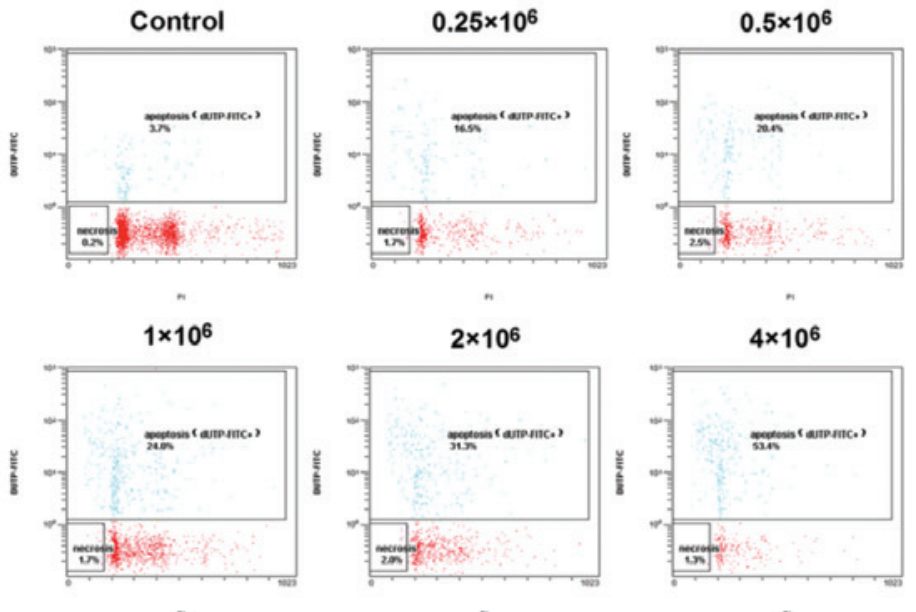

Figure 3. UC-MSCs induced the apoptosis of HepG2 cells. Using a Transwell system, UC-MSCs were co-cultured with HepG2 cells. A range of initial seeding densities for UC-MSCs was tested $\left(0.25 \times 10^{6}, 0.5 \times 10^{6}, 1 \times 10^{6}, 2 \times 10^{6}\right.$ or $4 \times 10^{6}$ cells/well). HepG2 cell apoptosis was determined using a terminal deoxynucleotidyl transferase-mediated nick end labeling assay at the indicated time points. The $\mathrm{x}$-axis indicates the incubation time (h). ${ }^{*} \mathrm{P}<0.01$ compared with $0.25 \times 10^{6}$ UC-MSCs incubation; ${ }^{~} \mathrm{P}<0.01$ compared with $24 \mathrm{~h}$ incubation. UC-MSCs, umbilical cord-derived mesenchymal stem cells. 


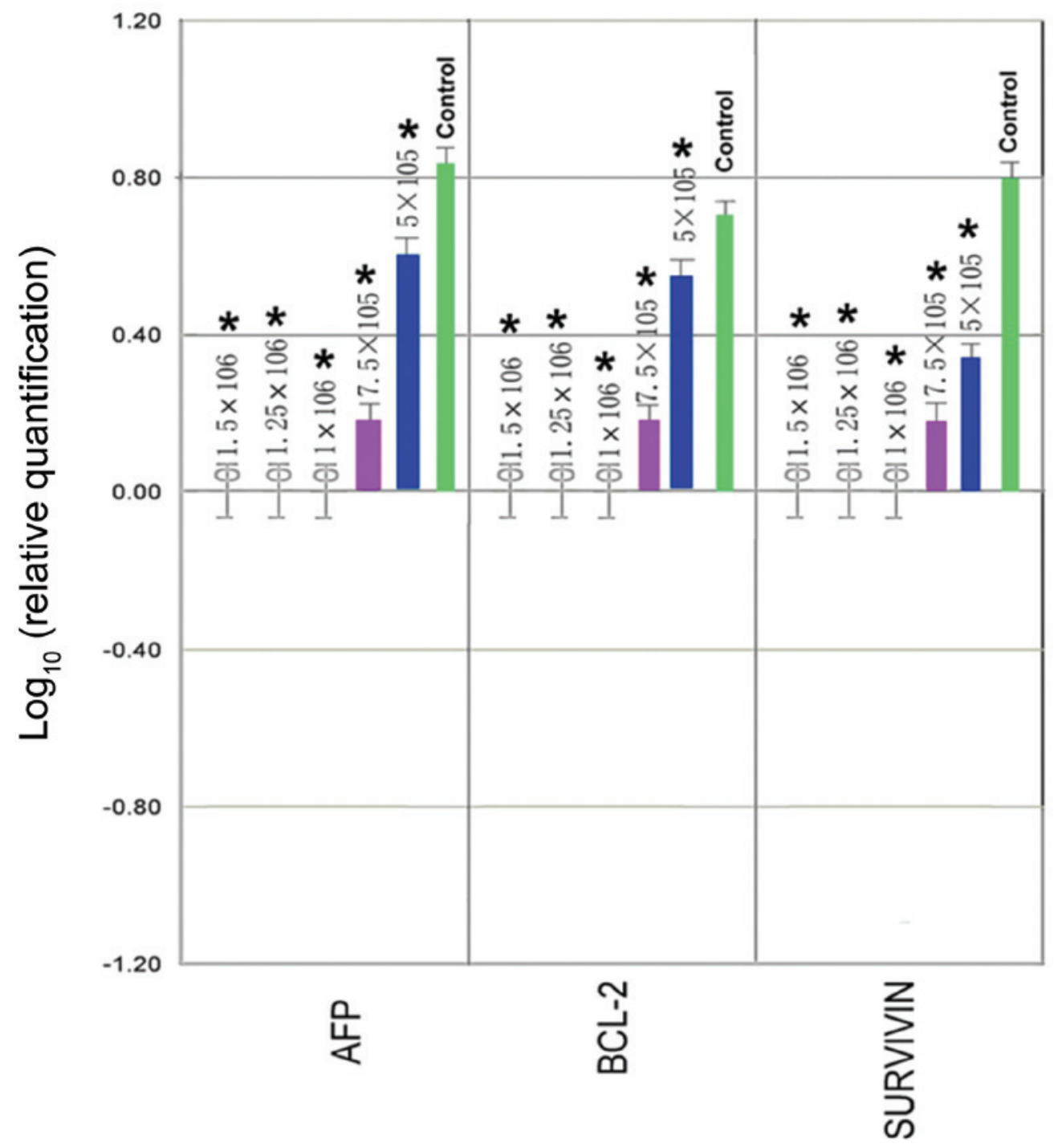

Figure 4. UC-MSCs downregulated the expression of AFP, Bcl-2 and Survivin mRNA in HepG2 cells after co-culture. HepG2 cells were co-cultured for $72 \mathrm{~h}$ with a range of UC-MSC initial seeding densities $\left(0.5 \times 10^{6}, 0.75 \times 10^{6}, 1 \times 10^{6}, 1.25 \times 10^{6}\right.$ or $1.5 \times 10^{6}$ cells/well). AFP, Bcl-2 and Survivin mRNA expression levels were detected by reverse transcription-quantitative polymerase chain reaction analysis. The x-axis indicates the density of cells initially seeded (cells/well) as well as the name of the genes examined. Data were calculated from three independent experiments. ${ }^{~} \mathrm{P}<0.01$ compared with the control. UC-MSCs, umbilical cord-derived mesenchymal stem cells; AFP, $\alpha$-fetoprotein.

typical phenotype of MSCs derived from the umbilical cord. No significant difference was observed in the expression of these biomarkers between passage three and passage seven UC-MSCs. These data identified the MSCs derived from the umbilical cord and passage seven to eight UC-MSCs were used for the remaining experiments.

Co-culture of UC-MSCs suppresses the proliferation of HepG2 cells. In order to investigate the effects of UC-MSCs on cultured HepG2 HCC cells, the two cell types were co-cultured using a Transwell system. Under normal conditions, cultured HepG2 cells were multi-polar and had a cobblestone-like appearance when they reached confluence (Fig. 2A). However, upon co-culture, the number of the HepG2 cells was reduced and cells displayed an irregular morphology. Additionally, HepG2 cell loss was accelerated when the initial seeding number of UC-MSCs was increased (Fig. 2A). The growth inhibition capability of UC-MSCs on HepG2 cells was further confirmed using an MTT assay. It was demonstrated that UC-MSCs time-dependently inhibited the proliferation of HepG2 cells $(\mathrm{P}<0.01$ for different incubation times; Fig. 2B). Enhanced proliferation inhibition was observed by increasing the initial seeding number of UC-MSCs into the Transwell compartment $(\mathrm{P}<0.01$ compared with different seeding densities). These results suggest that UC-MSCs can inhibit HepG2 cell proliferation.

Co-culture of UC-MSCs induces the apoptosis of HepG2 cells. The UC-MSC-induced apoptosis of HepG2 cells was investigated using a TUNEL assay. As shown in Fig. 3, co-culture of UC-MSCs induced the apoptosis of HepG2 cells in a time-dependent manner $(\mathrm{P}<0.01$ between different time points). Moreover, the initial seeding density of UC-MSCs also influenced the percentage of apoptotic HepG2 cells, as elevated apoptosis was detected with an increased number of UC-MSCs $(\mathrm{P}<0.01$ compared with different initial seeding cell number). It is important to note that over $50 \%$ of the HepG2 cells underwent apoptosis following a $72 \mathrm{~h}$ incubation 

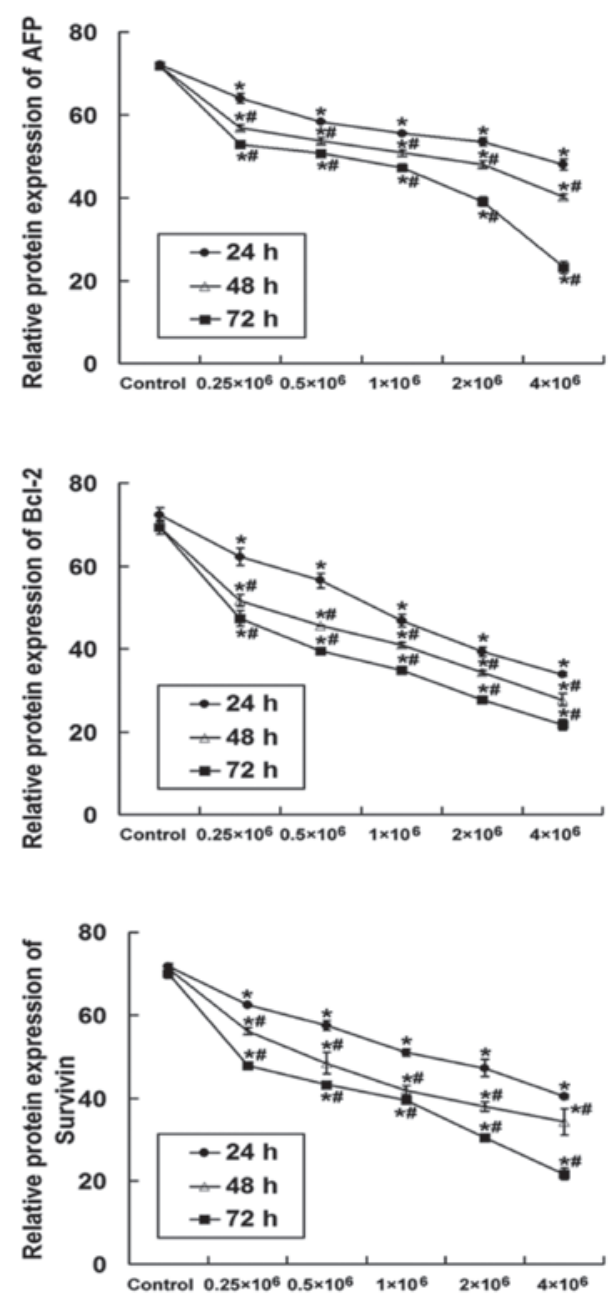

Figure 5. UC-MSCs downregulated AFP, Bcl-2 and Survivin protein expression in HepG2 cells after co-culture. HepG2 cells for 24,48 or $72 \mathrm{~h}$ were co-cultured with a range of initial seeding densities of UC-MSCs $\left(0.25 \times 10^{6}\right.$, $0.5 \times 10^{6}, 1 \times 10^{6}, 2 \times 10^{6}$ or $4 \times 10^{6}$ cells/well). HepG2 cells without co-culture were used as a control. AFP, Bcl-2 and Survivin protein expression levels were detected by flow cytometric analysis. The $\mathrm{x}$-axis indicates the density of cells initially seeded (cells/well). Data were calculated from three independent experiments. " $\mathrm{P}<0.01$ compared with control; ${ }^{\mathrm{P}} \mathrm{P}<0.01$ compared with $24 \mathrm{~h}$ of co-culture treatment. UC-MSCs, umbilical cord-derived mesenchymal stem cells; AFP, $\alpha$-fetoprotein.

with UC-MSCs when $4 \times 10^{6}$ UC-MSCs were seeded into the co-culture system. These data imply that UC-MSCs promote apoptosis in HepG2 cells.

Co-culture of UC-MSCs downregulates $m R N A$ and protein expression of AFP, Bcl-2 and Survivin mRNA in HepG2 cells. In order to understand the underlying molecular mechanism of UC-MSC-induced proliferation inhibition and apoptosis induction of HepG2 cells, mRNA and protein expression of AFP, Bcl-2 and Survivin in HepG2 cells were examined by RT-qPCR and flow cytometry, respectively. As shown in Fig. 4, AFP, Bcl-2 and Survivin mRNA expression were observed in control HepG2 cells. After $72 \mathrm{~h}$ of co-culture with UC-MSCs, the expression of these three genes in HepG2 cells was gradually reduced and the level of reduction was dependent on initial UC-MSC seeding density. At a low density of UC-MSCs $\left(0.5 \times 10^{6}\right.$ or $0.75 \times 10^{6}$ cells/well $)$, mRNA levels of
AFP, Bcl-2 and Survivin were reduced when compared with the control $(\mathrm{P}<0.01)$, whereas a high initial seeding density of UC-MSCs $\left(1 \times 10^{6}, 1.25 \times 10^{6}\right.$ or $1.5 \times 10^{6}$ cells/well $)$ essentially eliminated detection of AFP, Bcl-2 and Survivin expression.

To confirm the effects of UC-MSCs on AFP, Bcl-2 and Survivin expression in HepG2 cells, protein levels of these genes were analyzed by flow cytometric analysis after the indicated time period of co-culture. As shown in Fig. 5, co-culture of UC-MSCs significantly downregulated the protein levels of AFP, Bcl-2 and Survivin in HepG2 cells $(\mathrm{P}<0.01$ compared with control). Moreover, downregulation of protein levels appeared to be dependent on the initial seeding density of UC-MSCs, as a higher initial seeding density of UC-MSCs more efficiently decreased HepG2 AFP, Bcl-2 and Survivin protein expression $(\mathrm{P}<0.01)$. In addition, co-culture with UC-MSCs time-dependently reduced the expression of these proteins in HepG2 cells within $72 \mathrm{~h}$ of co-culture $(24 \mathrm{~h}$ vs. $48 \mathrm{~h}, 24 \mathrm{~h}$ vs. $72 \mathrm{~h}, 48 \mathrm{~h}$ vs. $72 \mathrm{~h} ; \mathrm{P}<0.01$ ). Therefore, it is possible that UC-MSCs may inhibit cell proliferation and promote apoptosis of HepG2 cells via regulating the expression of these proteins.

\section{Discussion}

Human MSCs, multipotent cells that can self-renew, proliferate and differentiate into a variety of cell types $(21,22)$, are emerging as novel cell-based delivery agents for cancer therapy (23). Nevertheless, the effect of MSCs on the growth, progression and metastasis of human malignancies, including HCC, remains controversial and the underlying molecular and cellular mechanisms are not yet fully elucidated.

Emerging lines of evidence suggest that MSCs are the potential precursor for tumor stroma (24). Other studies have shown that MSCs mediate the inhibition of tumor growth in vivo and in vitro (25-27). Application of genetically modified human MSCs, which express human pigment epithelium-derived factor, have been shown to inhibit HCC in nude mice, and thus it is has been suggested to be a promising approach for the treatment of HCC (28). Moreover, MSCs derived from the umbilical cord are hypothesized to be potent candidates for the clinical application of allogenic MSC-based therapies as they can easily be isolated from umbilical cord blood, cultured or modified in vitro, and autologously transplanted into patients, thus overcoming the difficulties associated with immune rejection of transplanted cells. Compared with the 'gold standard' of bone marrow MSCs, UC-MSCs showed a higher proliferative potential and were capable of osteogenic, chondrogenic and adipogenic differentiation $(29,30)$. Moreover, these cells have been shown to home to tumor tissues but not to healthy tissues and they do not form teratomas when injected into severecombined immunodeficiency mice (31).

In the present study, MSCs derived from the umbilical cord were identified and were characterized by expression of genetic and surface markers (positive for CD29, CD44, CD90 and CD105 and negative for CD34 and CD45). They appeared to be stable in terms of their surface marker expression in early passage (passages three to seven), which is in accordance with a previous study (32). Additionally, to the best of our knowledge, this study demonstrated for the first 
time, that UC-MSCs have the ability to inhibit the proliferation of HCC HepG2 cells in vitro, which suggests that MSCs from the umbilical cord exhibit antitumor properties. These data are consistent with previous studies, which showed antitumor effects of UC-MSCs on breast cancer $(15,33,34)$ and bronchioloalveolar carcinoma (35). It is possible that UC-MSCs may act in a paracrine manner through secretion of cytokines, interleukins and growth factors. A recent study identified several factors, including interleukins, fibroblast growth factor and insulin-like growth factor binding protein family members, that were secreted by UC-MSCs (36). However, the underlying mechanisms of these cytokines and factors remain unknown.

The potential role of UC-MSCs in promoting the apoptosis of HepG2 cells upon co-culture was also investigated. The results demonstrated that UC-MSCs significantly enhanced HepG2 apoptosis. AFP functions as a regulatory factor in tumor cell growth, as it acts as a protein-binding partner for caspase-3 and blocks apoptotic signaling in human AFP-producing hepatoma cells (37). Moreover, the level of AFP is positively correlated with another well-defined oncoprotein, Bcl-2, in patients with HCC (38). AFP may inhibit the translocation of the retinoic acid receptor (RAR)- $\beta$ into the nucleus through competition with all trans-retinoic acid for binding to RAR- $\beta$ and diminish the negative regulatory effect of RAR- $\beta$ on Survivin (39). These data indicate an association of AFP, Bcl-2 and Survivin with apoptosis inhibition. The present study also demonstrated that co-culture of UC-MSCs downregulated the expression of these three genes in HepG2 cells, suggesting UC-MSCs may promote apoptosis through regulating the expression of genes participating in apoptosis signaling.

Future studies will focus on investigating the underlying molecular mechanism involved in UC-MSC-mediated tumor growth inhibition and apoptosis. Accumulating evidence has also highlighted the tumor-promoting properties of MSCs (40-43). It has been shown that MSCs enhanced tumor growth but significantly inhibited the invasiveness and metastasis of HCC (13). This discrepancy, in relation to the present results, may be due to the different characteristics of bone marrow-derived MSCs used previously and the UC-MSCs used in this study. The invasiveness and metastatic activities of tumor cells following co-culture with UC-MSCs warrants further investigation.

In conclusion, the present study shows that UC-MSCs efficiently suppress the proliferation of HepG2 cells. In addition, UC-MSCs promote apoptosis through downregulation of AFP, Bcl-2 and Survivin gene expression, which are associated with apoptotic signaling pathways. These data provide valuable insights into the biological properties of UC-MSCs and suggest that UC-MSCs may be a promising cell source for MSC-based therapy for HCC.

\section{Acknowlegdements}

This study was supported by the National Natural Science Foundation of China (grant no. 81360072), the Natural Science Foundation of Yunnan Province (grant no. 2013FB050) and the Health Science and Technology Project of Yunnan Province (grant nos. 2014NS108 and 2014NS109).

\section{References}

1. Lau WY and Lai EC: Hepatocellular carcinoma: Current management and recent advances. Hepatobiliary Pancreat Dis Int 7: 237-257, 2008

2. Llovet JM, Burroughs A and Bruix J: Hepatocellular carcinoma. Lancet 362: 1907-1917, 2003.

3. Lai EC and Lau WY: The continuing challenge of hepatic cancer in Asia. Surgeon 3: 210-215, 2005.

4. Lau WY: Primary liver tumors. Semin Surg Oncol 19: 135-144, 2000.

5. Lau WY: Management of hepatocellular carcinoma. J R Coll Surg Edinb 47: 389-399, 2002.

6. Bruix J and Sherman M: Management of hepatocellular carcinoma: An update. Hepatology 53: 1020-1022, 2011.

7. Llovet JM, Schwartz M and Mazzaferro V: Resection and liver transplantation for hepatocellular carcinoma. Semin Liver Dis 25: 181-200, 2005.

8. Russo FP and Parola M: Stem and progenitor cells in liver regeneration and repair. Cytotherapy 13: 135-144, 2011.

9. Karahuseyinoglu S, Kocaefe C, Balci D, Erdemli E and Can A: Functional structure of adipocytes differentiated from human umbilical cord stroma-derived stem cells. Stem Cells 26: 682-691, 2008.

10. Campard D, Lysy PA, Najimi M and Sokal EM: Native umbilical cord matrix stem cells express hepatic markers and differentiate into hepatocyte-like cells. Gastroenterology 134: 833-848, 2008.

11. Fu YS, Shih YT, Cheng YC and Min MY: Transformation of human umbilical mesenchymal cells into neurons in vitro. J Biomed Sci 11: 652-660, 2004.

12. Ma L, Feng Xy, Cui BL, Law F, Jiang XW, Yang LY, Xie QD and Huang TH: Human umbilical cord Wharton's Jelly-derived mesenchymal stem cells differentiation into nerve-like cells. Chin Med J (Engl) 118: 1987-1993, 2005.

13. Li GC, Ye QH, Xue YH, Sun HJ, Zhou HJ, Ren N, Jia HL, Shi J, Wu JC, Dai C, et al: Human mesenchymal stem cells inhibit metastasis of a hepatocellular carcinoma model using the MHCC97-H cell line. Cancer Sci 101: 2546-2553, 2010.

14. Khakoo AY, Pati S, Anderson SA, Reid W, Elshal MF, Rovira II, Nguyen AT, Malide D, Combs CA, Hall G, et al: Human mesenchymal stem cells exert potent antitumorigenic effects in a model of Kaposi's sarcoma. J Exp Med 203: 1235-1247, 2006.

15. Ma Y, Hao X, Zhang S and Zhang J: The in vitro and in vivo effects of human umbilical cord mesenchymal stem cells on the growth of breast cancer cells. Breast Cancer Res Treat 133: 473-485, 2012.

16. Yang WZ, Zhang Y, Wu F, Min WP, Minev B, Zhang M, Luo XL, Ramos F, Ichim TE, Riordan NH and Hu X: Safety evaluation of allogeneic umbilical cord blood mononuclear cell therapy for degenerative conditions. J Transl Med 8: 75, 2010.

17. Wang L, Li H, Zhang Y, Santella RM and Weinstein IB: HINT1 inhibits beta-catenin/TCF4, USF2 and NFkappaB activity in human hepatoma cells. Int J Cancer 124: 1526-1534, 2009.

18. Livak KJ and Schmittgen TD: Analysis of relative gene expression data using real-timequantitative PCR and the 2(-Delta Delta C(T)) Method. Methods 25: 402-408, 2001.

19. Kulterer B, Friedl G, Jandrositz A, Sanchez-Cabo F, Prokesch A, Paar C, Scheideler M, Windhager R, Preisegger KH and Trajanoski Z: Gene expression profiling of human mesenchymal stem cells derived from bone marrow during expansion and osteoblast differentiation. BMC Genomics 8: 70, 2007.

20. Troyer DL and Weiss ML: Wharton's jelly-derived cells are a primitive stromal cell population. Stem Cells 26: 591-599, 2008.

21. Jiang Y, Jahagirdar BN, Reinhardt RL, Schwartz RE, Keene CD, Ortiz-Gonzalez XR, Reyes M, Lenvik T, Lund T, Blackstad M, et al: Pluripotency of mesenchymal stem cells derived from adult marrow. Nature 418: 41-49, 2002.

22. Mareschi K, Ferrero I, Rustichelli D, Aschero S, Gammaitoni L, Aglietta M, Madon E and Fagioli F: Expansion of mesenchymal stem cells isolated from pediatric and adult donor bone marrow. J Cell Biochem 97: 744-754, 2006.

23. Sasportas LS, Kasmieh R, Wakimoto H, Hingtgen S, van de Water JA, Mohapatra G, Figueiredo JL, Martuza RL, Weissleder R and Shah K: Assessment of therapeutic efficacy and fate of engineered human mesenchymal stem cells for cancer therapy. Proc Natl Acad Sci USA 106: 4822-4827, 2009. 
24. Studeny M, Marini FC, Dembinski JL, Zompetta C, Cabreira-Hansen M, Bekele BN, Champlin RE and Andreeff M: Mesenchymal stem cells: Potential precursors for tumor stroma and targeted-delivery vehicles for anticancer agents. J Natl Cancer Inst 96: 1593-1603, 2004.

25. Matsuzuka T, Rachakatla RS, Doi C, Maurya DK, Ohta N, Kawabata A, Pyle MM, Pickel L, Reischman J, Marini F, et al: Human umbilical cord matrix-derived stem cells expressing interferon-beta gene significantly attenuate bronchioloalveolar carcinoma xenografts in SCID mice. Lung Cancer 70: 28-36, 2010

26. Ohlsson LB, Varas L, Kjellman C, Edvardsen K and Lindvall M: Mesenchymal progenitor cell-mediated inhibition of tumor growth in vivo and in vitro in gelatin matrix. Exp Mol Pathol 75: 248-255, 2003

27. Zhu Y, Sun Z, Han Q, Liao L, Wang J, Bian C, Li J, Yan X, Liu Y, Shao $C$ and Zhao RC: Human mesenchymal stem cells inhibit cancer cell proliferation by secreting DKK-1. Leukemia 23: 925-933, 2009

28. Gao Y, Yao A, Zhang W, Lu S, Yu Y, Deng L, Yin A, Xia Y, Sun B and Wang X: Human mesenchymal stem cells overexpressing pigment epithelium-derived factor inhibit hepatocellular carcinoma in nude mice. Oncogene 29: 2784-2794, 2010.

29. Baksh D, Yao R and Tuan RS: Comparison of proliferative and multilineage differentiation potential of human mesenchymal stem cells derived from umbilical cord and bone marrow. Stem Cells 25: 1384-1392, 2007.

30. Jeong JA, Hong SH, Gang EJ, Ahn C, Hwang SH, Yang IH $\mathrm{Han} \mathrm{H}$ and Kim $\mathrm{H}$ : Differential gene expression profiling of human umbilical cord blood-derived mesenchymal stem cells by DNA microarray. Stem Cells 23: 584-593, 2005.

31. Rachakatla RS, Marini F, Weiss ML, Tamura M and Troyer D Development of human umbilical cord matrix stem cell-based gene therapy for experimental lung tumors. Cancer Gene Ther 14: 828-835, 2007.

32. Weiss ML, Medicetty S, Bledsoe AR, Rachakatla RS, Choi M, Merchav S, Luo Y, Rao MS, Velagaleti G and Troyer D: Human umbilical cord matrix stem cells: Preliminary characterization and effect of transplantation in a rodent model of Parkinson's disease. Stem Cells 24: 781-792, 2006.

33. Ayuzawa R, Doi C, Rachakatla RS, Pyle MM, Maurya DK, Troyer D and Tamura M: Naive human umbilical cord matrix derived stem cells significantly attenuate growth of human breast cancer cells in vitro and in vivo. Cancer Lett 280: 31-37, 2009.
34. Ganta C, Chiyo D, Ayuzawa R, Rachakatla R, Pyle M, Andrews G, Weiss M, Tamura M and Troyer D: Rat umbilical cord stem cells completely abolish rat mammary carcinomas with no evidence of metastasis or recurrence 100 days post-tumor cell inoculation. Cancer Res 69: 1815-1820, 2009.

35. Matsuzuka T, Rachakatla RS, Doi C, Maurya DK, Ohta N, Kawabata A, Pyle MM, Pickel L, Reischman J and Marini F: Human umbilical cord matrix-derived stem cells expressing interferon-beta gene significantly attenuate bronchioloalveolar carcinoma xenografts in SCID mice. Lung Cancer 70: 28-36, 2010.

36. Liu $\mathrm{CH}$ and Hwang SM: Cytokine interactions in mesenchymal stem cells from cord blood. Cytokine 32: 270-279, 2005.

37. Li M, Li H, Li C, Zhou S, Guo L, Liu H, Jiang W, Liu X, Li P, McNutt MA and Li G: Alpha fetoprotein is a novel protein-binding partner for caspase-3 and blocks the apoptotic signaling pathway in human hepatoma cells. Int J Cancer 124: 2845-2854, 2009.

38. Ali MA, Koura BA, El-Mashad N and Zaghloul MH: The Bcl-2 and TGF-beta1 levels in patients with chronic hepatitis $\mathrm{C}$, liver cirrhosis and hepatocellular carcinoma. Egypt J Immunol 11: 83-90, 2004.

39. Li M, Li H, Li C, Guo L, Liu H, Zhou S, Liu X, Chen Z, Shi S, Wei J, et al: Cytoplasmic alpha-fetoprotein functions as a co-repressor in RA-RAR signaling to promote the growth of human hepatoma Bel 7402 cells. Cancer Lett 285: 190-199, 2009.

40. Ke CC, Liu RS, Suetsugu A, Kimura H, Ho JH, Lee OK and Hoffman RM: In vivo fluorescence imaging reveals the promotion of mammary tumorigenesis by mesenchymal stromal cells. PLoS One 8: e69658, 2013.

41. Ljujic B, Milovanovic M, Volarevic V, Murray B, Bugarski D, Przyborski S, Arsenijevic N, Lukic ML and Stojkovic M: Human mesenchymal stem cells creating an immunosuppressive environment and promote breast cancer in mice. Sci Rep 3: 2298, 2013.

42. Xiao W, Mohseny AB, Hogendoorn PC and Cleton-Jansen AM: Mesenchymal stem cell transformation and sarcoma genesis. Clin Sarcoma Res 3: 10, 2013.

43. Zhang P, Dong L, Yan K, Long H, Yang TT, Dong MQ, Zhou Y, Fan QY and Ma BA: CXCR4-mediated osteosarcoma growth and pulmonary metastasis is promoted by mesenchymal stem cells through VEGF. Oncol Rep 30: 1753-1761, 2013. 